\title{
Avaliação clínica e polissonográfica do aparelho BRD no tratamento da Síndrome da Apneia Obstrutiva do Sono
}

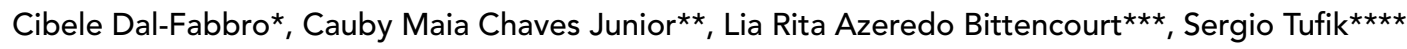

\section{Resumo}

Objetivos: este trabalho de pesquisa teve o intuito de realizar uma avaliação clínica e polissonográfica do efeito de um aparelho intraoral (AIO) para tratamento da Síndrome da Apneia Obstrutiva do Sono (SAOS), desenvolvido e testado por duas universidades federais brasileiras. Métodos: a amostra constou de 50 pacientes (idades entre 18 e 65 anos, sendo 33 homens e 17 mulheres) com diagnóstico polissonográfico inicial de SAOS de grau leve e moderado. Todos os pacientes submeteram-se a uma nova avaliação polissonográfica de noite inteira (em uso do AIO) aproximadamente 6 meses após a primeira avaliação. Baseado na diminuição dos eventos respiratórios obstrutivos, obtida com o uso do AIO, os pacientes foram então divididos em bons respondedores (redução de 50\% ou mais no índice de apneia e hipopneia (IAH), permanecendo abaixo de 10 eventos/hora) e maus respondedores (IAH permanecendo maior ou igual a 10 eventos/hora). Resultados e Conclusões: em 54\% da amostra o IAH diminuiu para menos de cinco eventos/hora com o uso do AIO; em 38\% a redução do IAH foi maior do que 50\%, mas permaneceu acima de cinco eventos/hora; e em $6 \%$ da amostra o IAH reduziu menos que 50\%. Os bons respondedores corresponderam a $86 \%$ da amostra estudada, enquanto os maus respondedores a 14\%. Houve melhora significativa na escala de sonolência, no IAH, nos microdespertares e na saturação mínima de oxihemoglobina com a terapia utilizada. O Índice de Massa Corpórea elevado parece interferir desfavoravelmente no desempenho do aparelho em estudo.

Palavras-chave: Apneia do Sono Tipo Obstrutiva. Polissonografia. Dispositivos de proteção respiratória. Ronco.

\footnotetext{
* Mestre em Reabilitação Oral-FOB-USP. Doutoranda em Medicina e Biologia do Sono na Unifesp.

** Mestre em Ortodontia pela Umesp e doutor em Ortodontia pela Unicamp. Pós-doutorando em Medicina e Biologia do Sono na Unifesp. Professor adjunto IV- Disciplina de Ortodontia- Universidade Federal do Ceará-UFC.

*** Professora adjunta de Medicina e Biologia do Sono na Unifesp.

$\star \star \star \star$ Livre-docente - professor titular de Medicina e Biologia do Sono na Unifesp.
} 


\section{INTRODUÇÃO E REVISÃO DE LITERATURA}

A Síndrome da Apneia Obstrutiva do Sono (SAOS) é um distúrbio respiratório relacionado ao sono caracterizado por repetidas obstruções parciais ou completas da via aérea superior durante o sono ${ }^{3,4}$. É uma das entidades clínicas mais comuns dentre os distúrbios do sono, afetando de 3 a $5 \%$ da população adulta ${ }^{30}$.

Dentre os achados clínicos clássicos estão o ronco intenso, intermitente, pausas respiratórias durante o sono, despertares recorrentes e ofegantes, sono não-reparador (fragmentado) e sonolência diurna excessiva ${ }^{3,5}$. O ronco entrecortado por paradas respiratórias é um relato típico das(os) companheiras(os) desses pacientes ${ }^{5,22}$. Entretanto, o ronco pode ocorrer na ausência de SAOS, caracterizando o ronco primário, o qual atinge cerca de $40 \%$ da população adulta masculina ${ }^{5}$.

O diagnóstico preciso dos distúrbios respiratórios do sono é dado por meio do exame de polissonografia (PSG), que permite quantificar os eventos acima relatados e a relação temporal desses com os parâmetros medidos durante uma noite inteira de sono. A quantificação dos eventos respiratórios por hora de sono, na forma do índice de apneia e hipopneia (IAH) confirma o diagnóstico e define a gravidade da SAOS: leve (entre 5 e 15), moderada (entre 15 e 30) e grave (acima de 30 eventos) ${ }^{3}$. Há ainda outros parâmetros que podem estar alterados nesses pacientes e podem ser visualizados na polissonografia, como: dessaturação de oxihemoglobina, alteração na porcentagem dos estágios de sono, redução na eficiência do sono e fragmentação do sono ${ }^{3,4,7}$.

A fisiopatologia da SAOS parece ser multifatorial, e há fatores anatômicos, funcionais e neuromusculares envolvidos. Os tecidos moles, o tecido adiposo, a musculatura e o esqueleto craniofacial afetam diretamente a configuração e a dimensão da faringe. Dessa forma, frequentemente observase pacientes com SAOS com hipotonia lingual, macroglossia, retrognatia mandibular e/ou maxilar, micrognatia, palato ogival, arcadas atrésicas e mordida cruzada ${ }^{10,18,22,25,27}$

Comumente, a SAOS está associada a complicações cardiovasculares e cognitivas como resultado da hipóxia intermitente e da fragmentação do sono, o que aumenta significativamente a morbidade e mortalidade dessa síndrome ${ }^{21}$. Estudos recentes estabelecem que a SAOS é um fator de risco independente para hipertensão arterial sistêmica (HAS) e contribui para a instalação e progressão de outras doenças cardiovasculares ${ }^{26}$. As consequências cognitivas estão relacionadas principalmente à atenção, memória e função executiva ${ }^{1,13}$

Como essa síndrome é uma condição duradoura e crônica, uma abordagem efetiva de tratamento se torna mandatória ${ }^{15}$. Os tratamentos da SAOS consistem de modalidades clínicas e cirúrgicas. A seleção da modalidade clínica de tratamento, seja com aparelhos de pressão positiva (CPAP, CPAP autoajustável, BiPAP) ou com os aparelhos intraorais (AIOs), está diretamente relacionada à gravidade da doença.

O CPAP nasal ("continuous positive airway pressure") é considerado o padrão-ouro no tratamento da $\operatorname{SAOS}^{14}$ (Fig. 1). Ele consiste de um método não-invasivo de aplicação de pressão positiva contínua de ar na via aérea, gerando um fluxo aéreo contínuo que, através de um tubo flexível, alcança uma máscara nasal ou nasobucal que é ajustada à face através de tiras fixadoras. Assim,

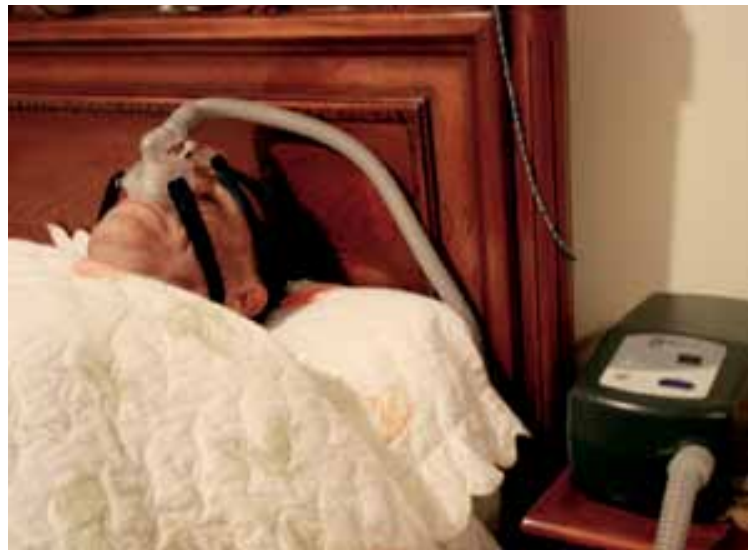

FIGURA 1 - CPAP ("continuous positive airway pressure"). 
cria-se no seu interior um coxim pneumático que tende a deslocar o palato mole em direção à base da língua, e a dilatar a área de secção de toda a faringe $\mathrm{e}^{13}$. Apesar de ser um tratamento extremamente eficaz, há problemas com a adesão ao uso dos aparelhos de pressão positiva e na sua aceitação em longo prazo.

Os Aparelhos Intraorais (AIOs) são dispositivos usados na cavidade oral durante o sono, com o objetivo de prevenir o colapso entre os tecidos da orofaringe e da base da língua, ou seja, a obstrução da via aérea superior ${ }^{2}$.

Os AIOs constituem uma forma de tratamento efetiva e bem aceita, e têm sido uma linha crescente de tratamento da SAOS e do ronco há mais de 20 anos. Hoje, há mais de 80 tipos de AIOs descritos, que se encaixam especialmente nas categorias de retentores linguais e reposicionadores mandibulares. Apenas alguns foram aprovados pelo FDA (Food and Drugs Administration) e há poucos com estudos controlados disponíveis. Há diferentes tipos de AIOs, com relação à fabricação (pré-fabricado ou confeccionado em laboratório), retenção, titulação da posição mandibular, abertura vertical anterior, liberdade de movimento mandibular e material de confecção, entre outros. A eficácia dos AIOs parece estar relacionada a alguns desses aspectos. Quando não se observa, durante o planejamento do dispositivo, os cuidados rela-

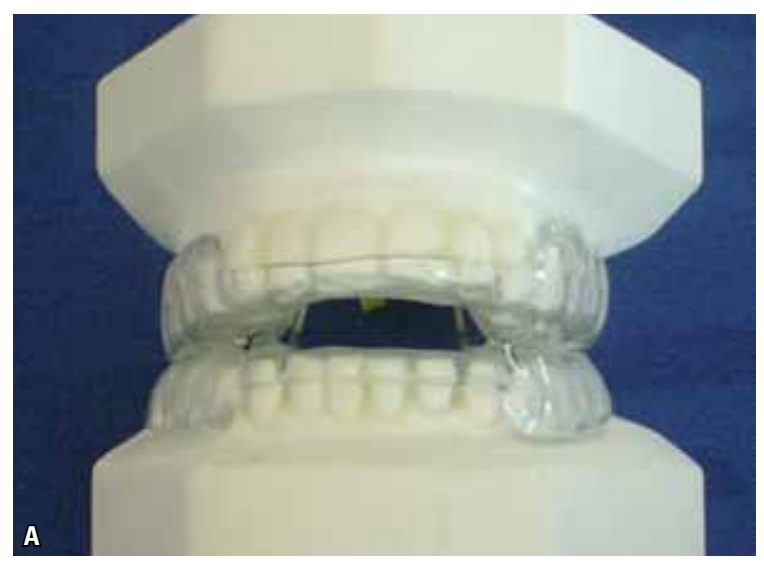

FIGURA 2 - AIO do tipo BRD: vistas anterior (A) e superior (B). cionados a esses fatores, temos maior probabilidade de efeitos colaterais, inclusive relacionados à DTM, às alterações oclusais, bem como à diminuição da adesão ao tratamento².

O tratamento com AIO está indicado aos pacientes com ronco primário e SAOS leve. Também nos casos de SAOS moderada ou grave quando houver intolerância ou recusa ao uso de CPAP, na contraindicação cirúrgica e como terapia substitutiva de curta duração $0^{7,17}$.

O objetivo do presente estudo foi avaliar o desempenho deste novo desenho de AIO para SAOS, buscando também comparar pacientes respondedores e não-respondedores quando se utiliza essa modalidade de tratamento.

\section{O APARELHO INTRAORAL}

O Brazilian Dental Appliance (BRD) foi desenvolvido a partir da experiência - dos pesquisadores da Odontologia na área de Medicina do Sono da Universidade Federal de São Paulo (Unifesp) e da Universidade Federal do Ceará (UFC) - com a utilização prévia de inúmeros outros dispositivos, principalmente norte-americanos e canadenses, muitos desses com limitações importantes, custos elevados e alguns sem estudos consistentes que respaldassem seu uso em pacientes com SAOS.

O BRD é um AIO reposicionador mandibular ajustável (Fig. 2A, B). É constituído de grampos

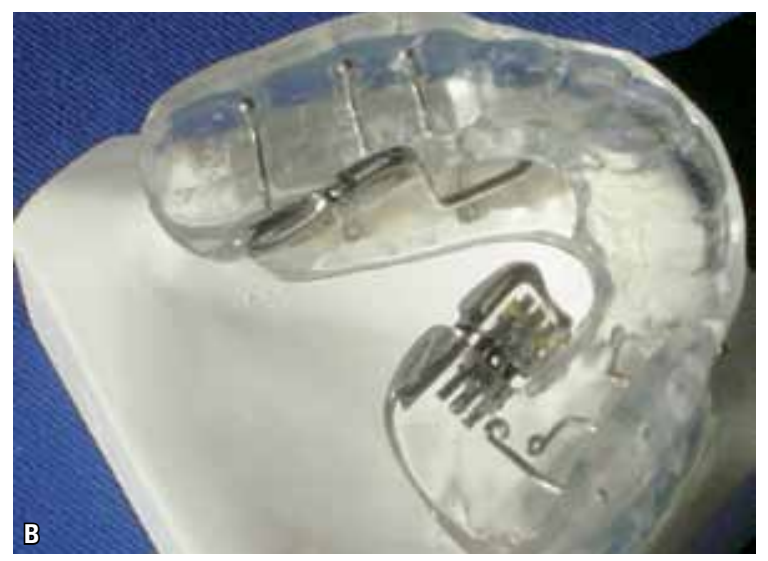


de retenção nos dentes posteriores e duas bases acrílicas de suporte (uma superior e outra inferior) recobrindo externa e internamente todos os dentes (anteriores e posteriores). Também possui dois mecanismos expansores (parafusos posicionados com seu longo eixo no sentido anteroposterior) independentes (um direito e outro esquerdo, posicionados na região posteropalatina da base acrílica superior). Desses mecanismos expansores saem duas hastes palatinas independentes (uma direita e outra esquerda) que se inserem inferiormente em dois pequenos tubos localizados na porção anterior (distal dos caninos inferiores) da base acrílica de suporte inferior. Este desenho proposto permite avanços sucessivos na posição mandibular, sem, no entanto, impedir movimentos mandibulares laterais. $\mathrm{Ou}$ seja, com o aparelho em posição, mesmo quando a mandíbula está mais anteriorizada, o paciente pode realizar movimentos laterais e pequena abertura bucal (Fig. 3).

\section{METODOLOGIA}

Esse estudo prospectivo incluiu 50 pacientes com SAOS indicados para o tratamento com AIO, seguindo o desenho do estudo (Fig. 4). Todos os pacientes haviam sido previamente diagnosticados através de um exame de polissonografia padronizada no departamento de Psicobiologia da Unifesp e na UFC.

Os critérios de inclusão consistiram de pacientes com SAOS leve ou moderada, idades entre 25 e 70 anos, com IMC (índice de massa corporal) menor que $30 \mathrm{Kg} / \mathrm{m}^{2}$ e com pelo menos 10 dentes em cada arcada dentária. Foram excluídos os pacientes submetidos previamente a procedimentos cirúrgicos para SAOS, usuários de CPAP, em uso corrente de qualquer droga que pudesse interferir na arquitetura do sono, trabalhadores de turno e, ainda, pacientes com alterações anatômicas importantes na via aérea superior, na forma de desvio de septo, hipertrofia de cornetos e/ou de amígdalas.

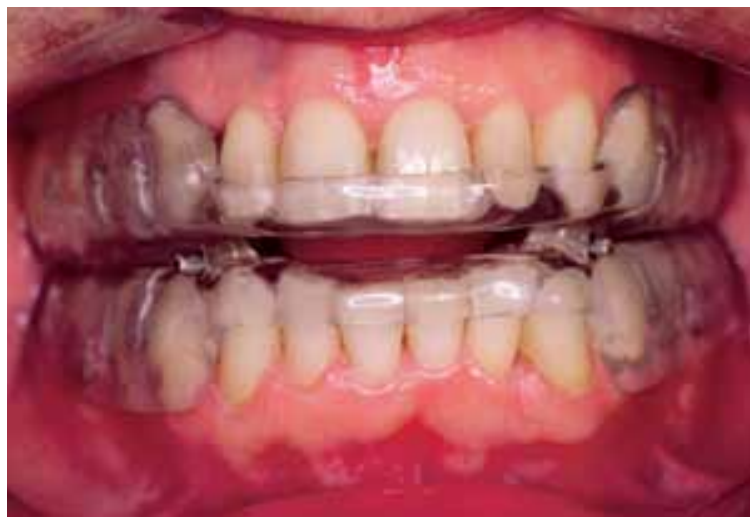

FIGURA 3 - BRD em boca (vista frontal).

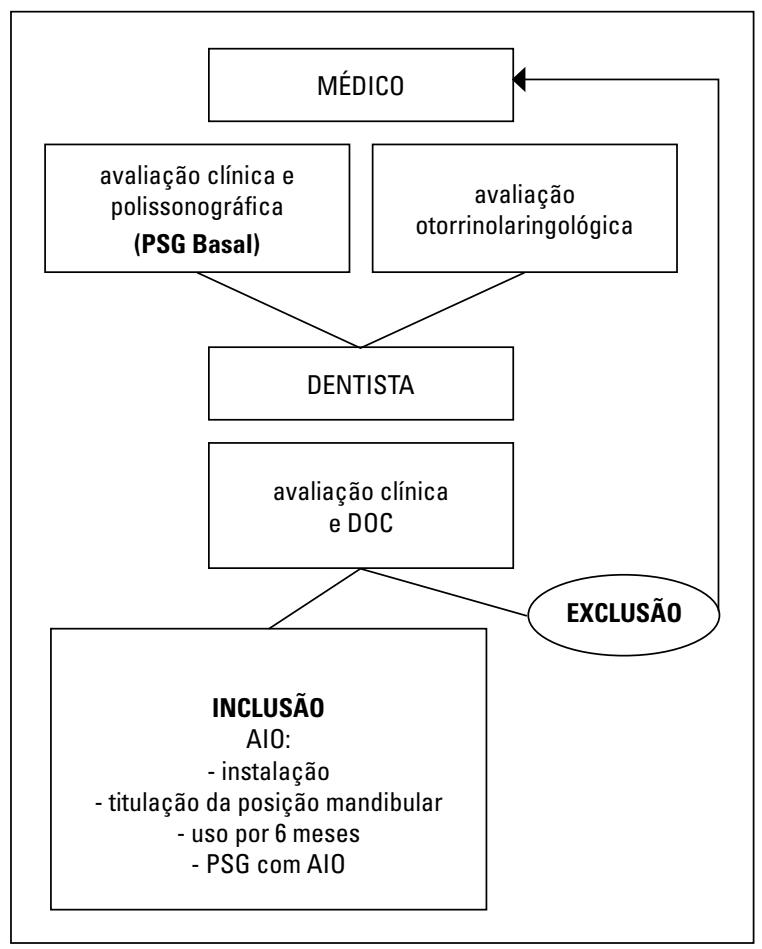

FIGURA 4 - Desenho do estudo.

Foram excluídos do estudo os voluntários que apresentaram diagnóstico de doença periodontal extensa, distúrbios graves nas articulações temporomandibulares ou unidades dentárias que tivessem anatomia insuficiente para a devida retenção do AIO. 


\section{Documentação odontológica (DOC)}

Todos os pacientes realizaram uma documentação ortodôntica básica, constando de radiografia panorâmica, telerradiografia de perfil, fotografias intra e extrabucais e modelos de estudo.

\section{Polissonografia (PSG)}

Todos os pacientes foram submetidos a uma polissonografia (PSG) basal para diagnóstico da SAOS e outra com o aparelho (BRD) na posição mandibular de máxima protrusão confortável. O tempo médio entre a primeira e a segunda polissonografia foi de seis meses.

A PSG de noite inteira foi feita em laboratório de sono usando um sistema contendo 13 canais, que incluíam eletroencefalograma, eletrocardiograma, eletro-oculograma, eletromiograma dos músculos submentoniano e tibial anterior, fluxo aéreo nasal e oral (medido com thermistor e/ou cânula nasal), movimentos torácicos e abdominais, posição corporal (decúbito) e saturação da oxihemoglobina, medida pela oximetria de pulso.

\section{Procedimentos clínicos e laboratoriais odontológicos}

Foram realizadas as moldagens das arcadas dentárias com alginato tipo II e imediatamente vazadas com gesso especial tipo IV. Obteve-se o registro da posição mandibular inicial com o dispositivo "George Gauge"2 e silicona de condensação (denso). Os modelos de gesso e o registro foram, então, utilizados para a confecção individualizada do aparelho BRD. Todos os aparelhos do estudo foram confeccionados em acrílico termopolimerizável, sendo a parte superior independente da inferior. A retenção foi obtida por grampos interproximais colocados entre todos os dentes posteriores. A ligação entre as duas arcadas foi feita por dois expansores posicionados na região palatina, logo abaixo da região cervical dos molares superiores. Esses expansores têm por objetivo possibilitar o avanço gradual da mandíbula em $11 \mathrm{~mm}$, em 44 incrementos de 0,25mm (Fig. 5).

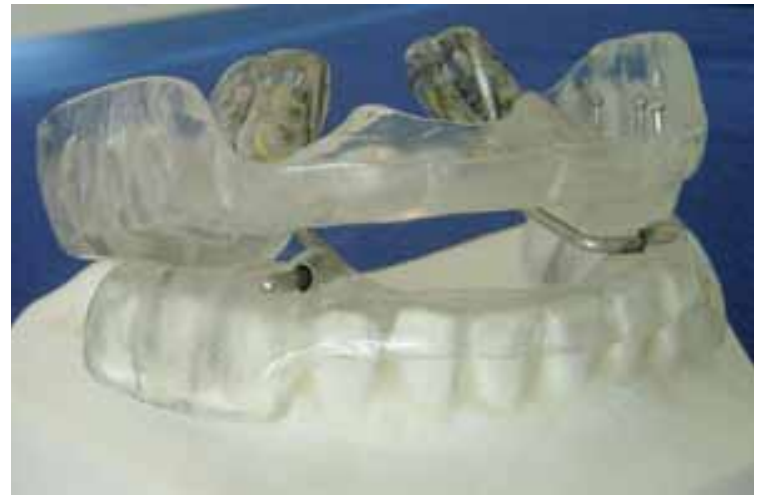

FIGURA 5 - Aparelho BRD utilizado no estudo.

Para obter a posição mandibular de máxima protrusiva confortável, ajustes progressivos foram feitos nos expansores dos aparelhos durante um período de 3 a 4 meses, de acordo com a presença de ronco e sintomas adicionais (sonolência, cansaço, sono fragmentado ou não-reparador).

O limite de $70 \%$ da protrusão mandibular máxima foi respeitado ${ }^{29}$. A cada consulta, os pacientes eram indagados sobre episódios de obstrução nasal e alteração de peso.

\section{Análise estatística}

Foi aplicado o teste de Kolmogorov-Smirnov para normalidade e, em seguida, o teste $t$ pareado para comparação entre a condição basal e com $\mathrm{AIO}$, quando os dados basais apresentaram distribuição normal, ou de Wilcoxon quando a distribuição foi não normal.

Subsequentemente, os pacientes foram divididos em dois grupos: bons e maus respondedores. Foi considerado um bom respondedor quando o IAH com AIO reduziu mais de 50\% em relação ao basal e foi menor do que 10 eventos por hora com AIO. Já o mau respondedor apresentava IAH com AIO maior ou igual a 10. Para essa situação, para comparar esses dois grupos, utilizou-se o teste $t$ de Student para amostras independentes, se a distribuição fosse normal, ou Mann-Whitney para distribuição não normal. 


\section{RESULTADOS}

Os dados são apresentados em média e desviopadrão. A amostra foi composta de 33 homens e 17 mulheres com idade média de 48,6 $\pm 12,3$ e IMC de $26,0 \pm 2,8 \mathrm{Kg} / \mathrm{m}^{2}$. A posição mandibular protrusiva final média foi de $8,8 \pm 1,3 \mathrm{~mm}$.

Os resultados da comparação entre a condição basal (inicial) e com AIO para todos os pacientes estão demonstrados na tabela 1 e, especificamente em relação ao número de apneias e hipopneias por hora de sono (IAH), ilustrados no gráfico 1. Nota-se melhora estatisticamente significativa em vários dos parâmetros estudados, como a diminuição da sonolência avaliada pela escala de Epworth, aumento na porcentagem de sono REM, diminuição do IAH, aumento na saturação mínima de oxihemoglobina, bem como a diminuição no número de microdespertares por hora de sono (Tab. 1).

Em 28 pacientes (56\% da amostra) o IAH com AIO normalizou, ou seja, esteve abaixo de 5 eventos/hora (sucesso); em 19 pacientes (38\% da amostra) o IAH ficou acima de 5 eventos/hora com o AIO, mas reduziu mais de $50 \%$ em relação a condição basal (sucesso parcial); e em 3 pacientes ( $6 \%$ da amostra) o IAH reduziu menos de 50\% em relação ao basal ou não reduziu (insucesso) (Quadro 1).

Considerando os resultados quanto ao IAH, os pacientes foram divididos em dois grupos: bons e maus respondedores ao tratamento. Foi considerado um bom respondedor quando o IAH com AIO reduziu mais de $50 \%$ em relação ao basal e foi menor que 10 eventos por hora $(n=43)$ e, do contrário, foi considerado como um mau respondedor (n = 7) (Tab. 2, 3). Podemos verificar na tabela 2 que houve melhora estatisticamente significativa em vários dos parâmetros polissonográficos avaliados quando estudados isoladamente o grupo de bons respondedores, mas é também importante salientar que mesmo o grupo de maus respondedores apresentou melhora significativa na sonolência, no IAH e no número de microdespertares durante o sono (Tab. 3).
TABELA 1 - Comparação entre os parâmetros basais e com AIO (BRD) (média \pm desvio-padrão).

\begin{tabular}{cccc} 
& BASAL $(\mathbf{n}=\mathbf{5 0})$ & BRD $(\mathbf{n}=\mathbf{5 0})$ & P \\
\hline IMC $\left(\mathrm{Kg} / \mathrm{m}^{2}\right)$ & $26,0 \pm 2,8$ & $26,1 \pm 2,8$ & $\mathrm{~ns}$ \\
E.S.Epw & $10,1 \pm 4,9$ & $5,6 \pm 3,5$ & $\mathrm{p}<0,05$ \\
\%SOL & $14,5 \pm 11,0$ & $16,6 \pm 11,2$ & $\mathrm{~ns}$ \\
\%REM & $16,0 \pm 6,1$ & $19,5 \pm 6,9$ & $\mathrm{p}<0,05$ \\
IAH & $19,7 \pm 10,3$ & $5,6 \pm 4,7$ & $\mathrm{p}<0,05$ \\
Microdespertares $/ \mathrm{h}$ & $19,7 \pm 10,3$ & $10,6 \pm 6,0$ & $\mathrm{p}<0,05$ \\
Sat 0 Média & $94,1 \pm 2,4$ & $94,5 \pm 1,9$ & $\mathrm{~ns}$ \\
Sat O $_{2}$ Mínima & $84,1 \pm 7,4$ & $87,5 \pm 4,4$ & $\mathrm{p}<0,05$
\end{tabular}

ns = não-significativo estatisticamente, IMC = índice de massa corporal $\left(\mathrm{kg} / \mathrm{m}^{2}\right)$, E.S.Epw = Escala de Sonolência de Epworth, \%SOL = \% sono de ondas lentas, $\%$ REM = \% sono REM, IAH = número de eventos de apneia e hipopneia por hora de sono, Sat $\mathrm{O}_{2}$ Média = saturação de oxihemoglobina média, Sat $\mathrm{O}_{2}$ Mínima = saturação de oxihemoglobina mínima.

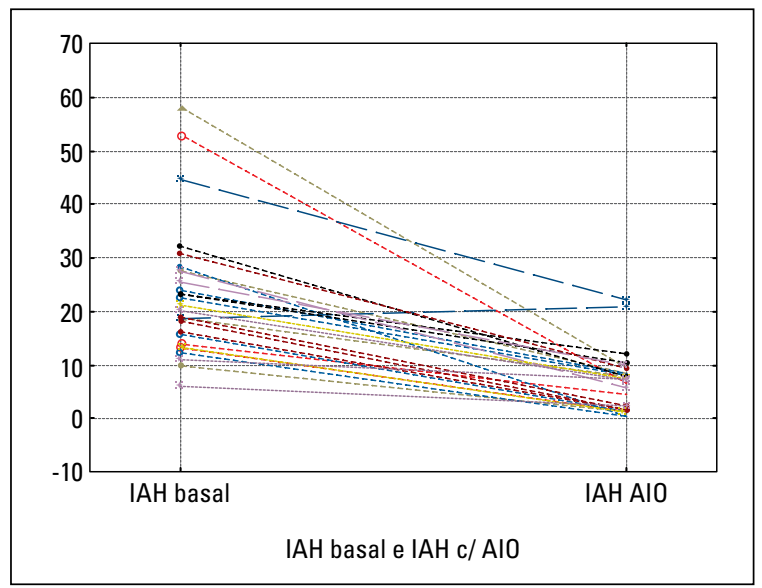

GRÁFICO 1 - IAH basal e IAH com AIO para todos ao pacientes.

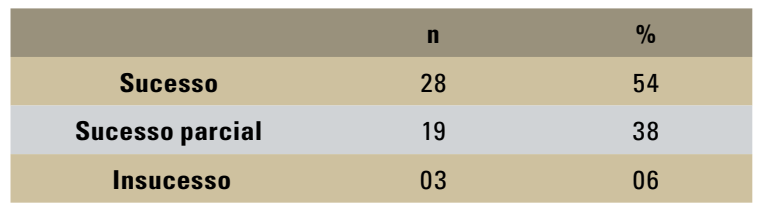

QUADRO 1 - Porcentagem de sucesso, sucesso parcial e insucesso para o total da amostra tratada com AIO (BRD).

A comparação dos resultados entre bons e maus respondedores demonstrou que aqueles que responderam mal ao tratamento apresentavam índice de massa corporal maior $(\mathrm{p}<0,05)$, inclusive com aumento de peso ao longo do tratamento (Tab. 4, Gráf. 2A, B). Por outro lado, outros dois parâmetros 
TABELA 2 - Comparação entre os parâmetros basais e com AIO (BRD) (média \pm desvio-padrão) no grupo de bons respondedores.

\begin{tabular}{cccc}
$\mathbf{n}=\mathbf{4 3}$ & BASAL & BRD & P \\
\hline IMC $\left(\mathrm{Kg} / \mathrm{m}^{2}\right)$ & $25,7 \pm 2,8$ & $25,8 \pm 2,8$ & $\mathrm{~ns}$ \\
E.S.EPW & $10,1 \pm 5,2$ & $5,3 \pm 3,5$ & $\mathrm{p}<0,05$ \\
\%SOL & $13,7 \pm 9,9$ & $15,4 \pm 10,9$ & $\mathrm{~ns}$ \\
\%REM & $15,9 \pm 6,4$ & $19,9 \pm 7,2$ & $\mathrm{p}<0,05$ \\
IAH & $19,2 \pm 10,2$ & $4,3 \pm 2,9$ & $\mathrm{p}<0,05$ \\
Microdesp./h & $19,7 \pm 10,5$ & $10,5 \pm 6,0$ & $\mathrm{p}<0,05$ \\
Sat $0_{2}$ Média & $94,1 \pm 2,5$ & $94,6 \pm 2,0$ & $\mathrm{~ns}$ \\
Sat O $_{2}$ Mínima & $83,8 \pm 7,8$ & $88,1 \pm 4,3$ & $\mathrm{p}<0,05$
\end{tabular}

ns = não-significativo estatisticamente, IMC = índice de massa corporal $\left(\mathrm{kg} / \mathrm{m}^{2}\right)$, E.S.Epw = Escala de Sonolência de Epworth, \%SOL = \% sono de ondas lentas, $\% \mathrm{REM}=\%$ sono REM, IAH = número de eventos de apneia e hipopneia por hora de sono, Sat $0_{2}$ Média = saturação de oxihemoglobina média, Sat $\mathrm{O}_{2}$ Mínima= saturação de oxihemoglobina mínima.
TABELA 3 - Comparação entre os parâmetros basais e com AIO (BRD) (média \pm desvio-padrão) no grupo de maus respondedores.

$\begin{array}{cccc}\mathbf{n}=\mathbf{0 7} & \text { BASAL } & \text { BRD } & \text { P } \\ \text { IMC }\left(\mathrm{Kg} / \mathrm{m}^{2}\right) & 27,7 \pm 2,1 & 28,2 \pm 2,3 & \mathrm{~ns} \\ \text { E.S.EPW } & 10,4 \pm 3,0 & 7,2 \pm 3,3 & \mathrm{p}<0,05 \\ \text { \% SOL } & 19,4 \pm 16,7 & 23,9 \pm 11,2 & \mathrm{~ns} \\ \text { \%REM } & 16,4 \pm 4,3 & 16,8 \pm 3,7 & \mathrm{~ns} \\ \text { IAH } & 22,9 \pm 10,9 & 13,9 \pm 5,3 & \mathrm{p}<0,05 \\ \text { Microdesp./h } & 19,7 \pm 9,2 & 11,6 \pm 6,9 & \mathrm{p}<0,05 \\ \text { Sat } 0_{2} \text { Média } & 94,3 \pm 1,6 & 94,4 \pm 1,6 & \mathrm{~ns} \\ \text { Sat } 0_{2} \text { Mínima } & 85,7 \pm 4,5 & 83,7 \pm 2,6 & \mathrm{~ns}\end{array}$

ns = não-significativo estatisticamente, IMC = índice de massa corporal $\left(\mathrm{kg} / \mathrm{m}^{2}\right)$, E.S.Epw = Escala de Sonolência de Epworth, \%SOL = \% sono de ondas lentas, $\%$ REM = \% sono REM, IAH = número de eventos de apneia e hipopneia por hora de sono, Sat $0_{2}$ Média = saturação de oxihemoglobina média, Sat $\mathrm{O}_{2}$ Mínima = saturação de oxihemoglobina mínima.
TABELA 4 - Comparação entre bons e maus respondedores.

\begin{tabular}{cccc} 
& $\begin{array}{c}\text { BONS } \\
\text { RESPONDEDORES }\end{array}$ & $\begin{array}{c}\text { MAUS } \\
\text { RESPONDEDORES }\end{array}$ & P \\
\hline IDADE & $47,5 \pm 11,6$ & $55,3+15,4$ & $\mathrm{~ns}$ \\
\hline IMC Basal & $25,7 \pm 2,8$ & $27,7 \pm 2,1$ & $\mathrm{p}<0,05$ \\
\hline IMC c/ AIO & $25,8 \pm 2,8$ & $28,2 \pm 2,3$ & $\mathrm{p}<0,05$ \\
$\begin{array}{c}\text { E.S.EPW } \\
\text { Basal }\end{array}$ & $10,1 \pm 5,2$ & $10,4 \pm 3,0$ & $\mathrm{~ns}$ \\
$\begin{array}{c}\text { IAH Basal } \\
\text { Microdesp. } \\
\text { Basal }\end{array}$ & $19,2 \pm 10,2$ & $22,9 \pm 10,9$ & $\mathrm{~ns}$ \\
$\begin{array}{c}\text { Sat 0 } \\
\text { Média Basal }\end{array}$ & $94,7 \pm 10,5$ & $19,7 \pm 9,2$ & $\mathrm{~ns}$ \\
$\begin{array}{c}\text { Sat 0 } \\
\text { Mín. Basal }\end{array}$ & $83,8 \pm 7,8$ & $85,7 \pm 4,5$ & $\mathrm{~ns}$ \\
$\begin{array}{c}\text { Avanço AI0 } \\
\text { (mm) }\end{array}$ & $8,8 \pm 1,3$ & $8,6+1,9$ & $\mathrm{~ns}$ \\
\hline
\end{tabular}

ns = não-significativo estatisticamente.

apresentaram tendência a diferença, embora não estatisticamente significativa, como a idade (maior nos maus respondedores) e a gravidade da SAOS (mais grave nos maus respondedores), sendo essa não-significativa para IAH, nem para saturação de oxihemoglobina. Vale ressaltar que a posição final (protrusiva) com o AIO não foi diferente entre respondedores e não-respondedores (Tab. 4).
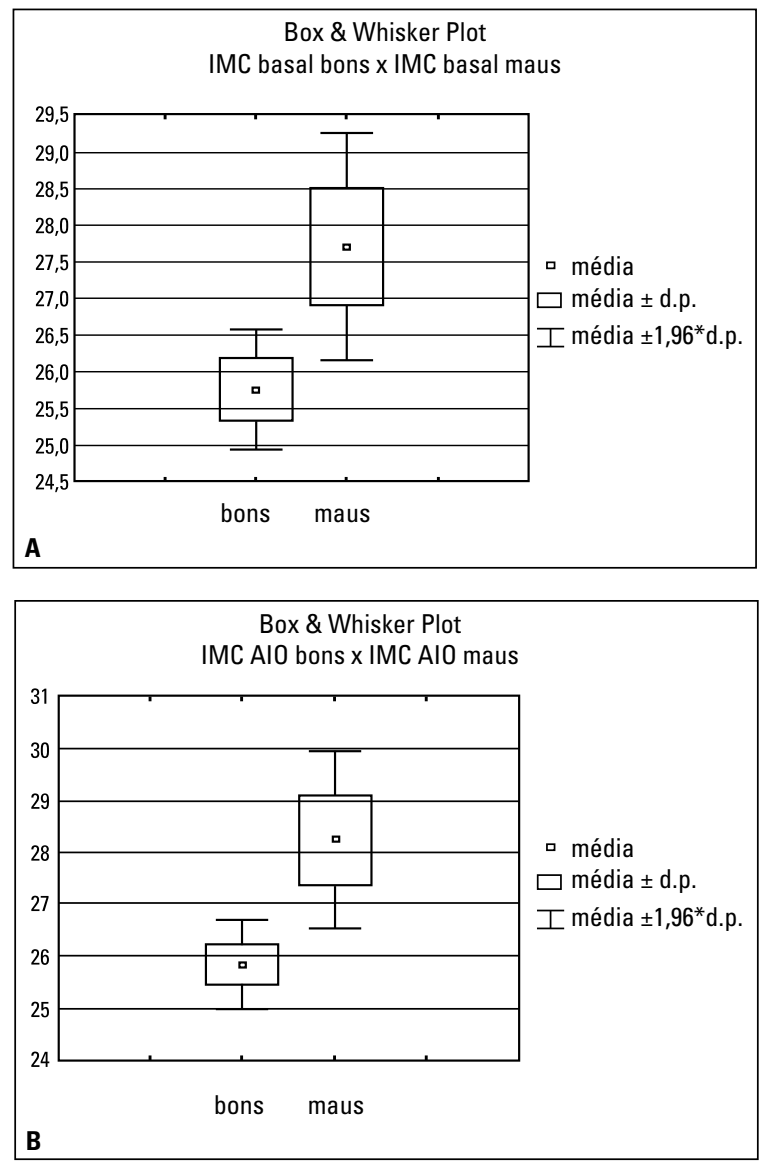

GRÁFICO 2 - A) IMC basal para os grupos bons e maus respondedores. B) IMC com AIO para os 2 grupos. 


\section{DISCUSSÃO}

Os reposicionadores mandibulares para tratamento da SAOS e do ronco podem ser de dois subtipos: ajustáveis e não-ajustáveis. Essa última categoria está caindo em desuso pela dificuldade de trabalho e pior adaptação por parte dos pacientes. Dos AIOs, os que parecem ter maior eficácia são os de avanço mandibular progressivo ou ajustáveis, que apresentam ótima retenção tanto à maxila quanto à mandíbula, além de serem de tamanho reduzido para aumentar o conforto ${ }^{8}$.

A diferença do BRD em relação aos demais aparelhos é a contenção da abertura bucal e o fato de haver dois elementos de ligação entre as arcadas (dois mecanismos expansores) posicionados na parte interna da arcada superior. Aparelhos que possuem retenção apenas na maxila, não conseguem manter uma posição mandibular estável ${ }^{2}$, permitindo a abertura bucal durante o sono e um giro mandibular no sentido horário, que pode influenciar desfavoravelmente as dimensões faringeanas. $\mathrm{O}$ fato de serem dois expansores independentes faz com que o aparelho posicione a arcada inferior de forma mais estável, propiciando a posição anterior sem permitir muita abertura bucal. Quando se faz esses aparelhos com um único expansor, permite-se maior abertura, o que não proporciona posição mandibular estável, possibilitando que a língua obstrua a passagem do fluxo aéreo superior. Além disso, um único expansor não permite avanços mandibulares assimétricos. Quando se faz aparelhos com dois expansores posicionados externamente às arcadas, têm-se limitações importantes à liberdade mandibular no sentido lateral e há possibilidade de injúrias à mucosa jugal.

Esse novo desenho, ao incorporar dois expansores internamente, permite ativações assimétricas, quando necessário, sem impedir pequenos movimentos laterais mandibulares durante o sono. Além disso, não causa injúrias ao dorso da língua.

$\mathrm{O}$ desenho do BRD foi desenvolvido com o objetivo de solucionar esses pontos, pois o aparelho propicia uma posição mandibular estável tan- to no sentido vertical (abertura) como no sentido anteroposterior (protrusão mandibular). Também promove maior conforto, sendo extremamente versátil quanto à possibilidade de avanços mandibulares progressivos, individualizados e mensuráveis, tornando seu uso mais fisiológico.

Os resultados de 30 diferentes estudos, em 456 pacientes com SAOS e 224 com ronco, durante um período de 10 anos (1982-1992), mostram que aparelhos intraorais têm o potencial para um significativo aumento da via aérea com redução média dos índices de SAOS em 54,3\% dos casos e melhora do ronco em 87,5\% ${ }^{23}$. Em 1995, Nowara et al. ${ }^{20}$ selecionaram 21 publicações e revisaram os resultados de 320 pacientes tratados de SAOS e ronco com AIOs. Observaram que o ronco foi totalmente eliminado em quase todos os pacientes e, nos que não obtiveram esse resultado, houve uma grande melhora. Quanto à SAOS, houve uma melhora de $60 \%$ do índice de apneia-hipopneia IAH, em média (IAH pré = 47 e pós = 19), e aproximadamente a metade dos pacientes atingiu IAH $<10$, que é considerado como sucesso por muitos autores ${ }^{20}$. Liu et al. ${ }^{19}$ observaram redução média do IAH de 40,3 para 17,1 e uma melhora média não-significativa da $\mathrm{SaO}_{2}$ de $76 \%$ para $80 \%$ com o aparelho Klearway ${ }^{\mathrm{TM}}$. Entretanto, nota-se que esses resultados não são uniformes, com pacientes respondendo bem rapidamente à terapia e outros em que os resultados não são tão animadores. Isso pode ser atribuído à falta de padronização dos estudos, principalmente em relação às amostras selecionadas e à forma de avaliação dos resultados.

No presente estudo, houve o cuidado de selecionar aqueles indivíduos que realmente tinham indicação para terapia com AIO, e o acompanhamento dos indivíduos seguiu as diretrizes atualizadas do Consenso Brasileiro de Ronco e Apneia ${ }^{7}$. Dessa forma, a avaliação dos resultados envolveu aspectos polissonográficos em relação aos parâmetros respiratórios (como o índice de apneia e hipopneia), mas também em relação à arquitetura do sono (como porcentagem de sono de 
ondas lentas e de sono REM). Critérios clínicos validados e extremamente importantes, como a escala de sonolência de Epworth, também foram utilizados. Observou-se melhora estatisticamente significativa em vários dos parâmetros avaliados, como: a diminuição da sonolência através da escala de Epworth, aumento na porcentagem de sono REM, diminuição do índice de apneia e hipopneia, aumento na saturação mínima de oxihemoglobina, bem como a diminuição no número de microdespertares por hora de sono (Tab. 1). Esses achados corroboram com as pesquisas de Bernhold e Bondemark ${ }^{6}$ que relataram uma melhora da $\mathrm{SaO}_{2}$ e uma diminuição da sonolência diurna na maioria dos seus pacientes com AIO. Observou-se uma melhora da qualidade do sono dos pacientes, com aumento da porcentagem do estágio REM, mas pequena influência sobre o tempo de sono NREM, conforme encontrado também por Henke, Frantz e Kuna ${ }^{16}$. Entretanto, Rose et al. ${ }^{24}$ relataram que a arquitetura do sono não mudou significativamente durante seu estudo com o AIO do tipo ativador Karwetzky.

Em relação aos eventos obstrutivos por hora de sono (IAH), os resultados demonstraram que, em 56\% da amostra, o IAH com AIO normalizou, ou seja, esteve abaixo de 5 eventos/hora (sucesso); em 38\% da amostra o IAH ficou acima de 5 eventos/hora com o AIO, mas reduziu mais de $50 \%$ em relação à condição basal (sucesso parcial); e em $6 \%$ da amostra o IAH reduziu menos de $50 \%$ em relação ao basal ou não reduziu (insucesso). Geralmente a eficácia é relatada baseando-se numa melhora nos índices de 50\% a 80\% daqueles obtidos inicialmente, mas ainda existe uma controvérsia grande em como padronizar os índices de sucesso ou mesmo a eficácia da terapia ${ }^{19}$.

Quando separados os pacientes em bons respondedores (IAH com AIO reduzido mais de 50\% e menor que 10 eventos por hora) e maus respondedores ao tratamento (IAH com AIO igual ou acima de 10 eventos/hora), verificou-se que 43 pacientes se enquadravam no primeiro grupo e
7 no segundo grupo. Melhoras significativas e em maior número de parâmetros avaliados foi detectada no grupo de bons respondedores (Tab. 2), mas vale ressaltar que, mesmo no grupo de maus respondedores, foram observadas melhoras significativas na sonolência, no IAH e no número de microdespertares durante o sono (Tab. 3).

Liu et al. ${ }^{19}$ estudaram pacientes com SAOS dividindo-os em três grupos de acordo com o grau de melhora no IAH: o grupo com resposta boa (redução do IAH > 75\%), o grupo com resposta moderada (redução do IAH entre 25 a 75\%) e o grupo com resposta pobre (redução do IAH $<25 \%$ ) em relação ao tratamento com aparelhos intraorais. Esse estudo demonstrou que havia uma diferença significativa na idade entre o grupo com resposta pobre e os outros grupos. Nos pacientes mais velhos e com maior IMC, o AIO foi menos efetivo. $\mathrm{O}$ estudo aqui apresentado também encontrou um índice de massa corporal maior, inclusive com aumento de peso ao longo do tratamento (Tab. 4, Gráf. 2A, B), nos pacientes que responderam mal ao tratamento com AIO. Parece também haver uma tendência de maior idade no grupo de maus respondedores.

Um aspecto importante é que a posição final (protrusiva) com o AIO não foi diferente entre respondedores e não-respondedores. Isso pressupõe que não são avanços mandibulares extremados e sem critério que irão melhorar a eficácia dos aparelhos intraorais. Incrementos exagerados na quantidade de avanço mandibular parecem não ter grande influência na melhora da SAOS ${ }^{29}$.

Os aparelhos intraorais oferecem o mais prático e lógico meio de se iniciar um tratamento para a maioria dos casos de SAOS. Um aparelho bem indicado, adequadamente confeccionado e controlado periodicamente, tem efetividade, não representa grandes despesas para os pacientes, é facilmente aceito pela maioria deles, além de poder ser associado a outras modalidades de tratamento ${ }^{9,12}$. O aparelho testado nesse estudo alcançou resultados bastante satisfatórios, sendo mais uma 
alternativa de aparelho reposicionador mandibular para tratamento de pacientes com apneia do sono do tipo obstrutiva.

\section{CONCLUSÃO}

Com a terapia utilizada houve melhora significativa na escala de sonolência, no IAH, nos microdespertares e na saturação mínima de oxihemoglobina. O Índice de Massa Corpórea elevado parece interferir desfavoravelmente no desempe- nho do aparelho em estudo, sugerindo que pacientes mais obesos ou que ganham peso ao longo do tratamento podem se tornar o grupo de insucesso para essa abordagem terapêutica da SAOS.

\title{
Clinical and polysomnographic assessment of Obstructive Sleep Apnea Syndrome treatment with BRD appliance
}

\begin{abstract}
Objectives: The current investigation aimed to carry out a clinical and polysomnographic assessment of treatment of Obstructive Sleep Apnea Syndrome (OSAS) with an oral appliance (OA) developed and tested by two Brazilian federal universities. Methodology: The sample was composed of 50 patients (aged between 18 and 65 years, 33 men and 17 women) with initial polysomnographic diagnosis of light to moderate OSAS. All patients underwent a second, full-night polysomnography with the use of the OA approximately 6 months after the first assessment. Based on the reduction of respiratory events obtained with the OA, patients were distributed in good responders (Apnea and Hypopnea Index/AHI under 10 and with reduction of at least $50 \%$ in relation to baseline); and poor responders (AHI of 10 or over with $\mathrm{OA}$ ). Results and Conclusion: In 54\% of the sample, AlH reduced to less than five events/hour with $\mathrm{OA}$; in $38 \%$ the $\mathrm{AHI}$ reduction was more than $50 \%$ in relation to baseline (but more than five); and in $6 \%$ of the sample, the AHI reduced less than $50 \%$. Good responders corresponded to $86 \%$ of the studied sample, while poor responders to $14 \%$. We noticed significant improvement in somnolence, in $\mathrm{AlH}$, in microarousals and also in minimum oxygen saturation with the treatment. Increased body mass index (BMI) seemed to interfere unfavorably in the performance of the OA studied.
\end{abstract}

Keywords: Sleep apnea. Obstructive. Respiratory protective devices. Polysomnography. Snoring.

\section{REFERÊNCIAS}

1. Adams N, Strauss M, Schluchter M, Redline S. Relation of measures of sleep-disordered breathing to neuropsychological functioning. Am J Respir Crit Care Med. 2001 Jun;163(7):162631.

2. Almeida FR, Dal-Fabbro C, Chaves Jr CM. Síndrome da apnéia e hipopnéia obstrutiva do sono (SAHOS): tratamento com aparelhos intra-orais. In: Tufik, S. Medicina e biologia do sono. $1^{a}$ ed. São Paulo: Manole; 2008. p. 263-80.

3. American Academy of Sleep Medicine. Sleep-related breathing disorders in adults: recommendations for syndrome definition and measurement techniques in clinical research. The Report of an American Academy of Sleep Medicine Task Force. Sleep. 1999 Aug;22(5):667-89.
4. American Academy of Sleep Medicine. International classification of sleep disorders. 2nd ed. Diagnostic and coding manual. Westchester: Illinois; 2005

5. Bassiri AG, Guilleminault C. Clinical features and evaluation of obstructive sleep apnea-hypopnea syndrome. In: Kryger MH, Roth T, Dement WC. Principles and Practice of Sleep Medicine. 3rd ed. Philladelphia: W.B. Saunders; 2000. p. 869-78.

6. Bernhold M, Bondemark L. A magnetic appliance for treatment of snoring patients with and without obstructive sleep apnea. Am J Orthod Dentofacial Orthop. 1998 Feb;113(2):144-55.

7. Bittencourt LRA. Diagnóstico e tratamento da Síndrome da Apnéia Obstrutiva do Sono (SAOS)-Guia Prático. $1^{\text {a }}$ ed. São Paulo: Médica Paulista; 2008 
8. Chaves CM Jr, Dal-Fabbro C, Almeida FR. Aparelhos intrabucais de avanço mandibular para tratamento da apnéia obstrutiva do sono. In: Dominguez GC. Nova visão em Ortodontia e Ortopedia Funcional dos Maxilares. 1 $^{\text {a }}$ ed. São Paulo: Ed. Santos; 2007. p. 355-60.

9. Clark GT, Nakand M. Dental appliances for the treatment of obstructive sleep apnea. J Am Dent Ass. 1989; 118:611-9.

10. Davies SF, Iber C. Obstructive sleep apnea associated with adult-acquired micrognathia from rheumatoid arthritis. Am Rev Respir Dis. 1983 Feb;127(2):245-7.

11. George PT. A new instrument for functional appliance bite registration. J Clin Orthod. 1992 Nov;26(11):721-3.

12. George PT. Treatment of snoring and obstructive sleep apnea with a dental device. Gen Dent. 1993 Jul/Aug;41(4):294-8.

13. Gozal D, Kheirandish L. Sleepiness and neurodegeneration in sleep-disordered breathing: convergence of signaling cascades. Am J Respir Crit Care Med. 2005 Jun 15;171(12):13257.

14. Grunstein R, Sullivan C. Continuous positive airway pressure for sleep breathing disorders. In: Kryger MH, Roth T, Dement WC. Principles and practice of sleep medicine. 3rd ed. Philladelphia: W.B. Saunders; 2000. p. 894-912.

15. Guilleminault C, Simmons FB, Motta J, Cummiskey J, Rosekind M, Schroeder JS, Dement WC. Obstructive sleep apnea syndrome and tracheostomy. Long-term follow-up experience. Arch Intern Med. $1981 \mathrm{Jul} ; 141(8): 985-8$.

16. Henke KG, Frantz DE, Kuna ST. An oral elastic mandibular advancement device for obstructive sleep apnea. Am J Respir Crit Care Med. 2000 Feb;161(2 Pt 1):420-5.

17. Kushida CA, Morgenthaler TI, Littner MR, Alessi CA, Bailey D, Coleman J Jr, Friedman L, Hirshkowitz M, Kapen S, Kramer M, Lee-Chiong T, Owens J, Pancer JP. Practice parameters for the treatment of snoring and obstructive sleep apnea with oral ap pliances: An update for 2005. An American Academy of Sleep Medicine Report. Sleep. 2006 Feb;29(2):240-3.

18. Li KK, Riley RW, Powell NB, Kushida C, Troell RJ. Obstructive sleep apnea syndrome in the Asian patient. Annual meeting of the American Academy of Otolaryngology-Head and Neck Surgery Foundation. New Orleans (LA); 1999.

19. Liu Y, Lowe AA, Fleetham JA, Park YC. Cephalometric and physiologic predictors of the efficacy of an adjustable oral appliance for treating obstructive sleep apnea. Am J Orthod Dentofacial Orthop. 2001 Dec;120(6):639-47.
20. Nowara WS, Lowe A, Wiegand L, Cartwright R. Oral appliances for the treatment of snoring and obstructive sleep apnea: a review. An Amer Sleep Disorders Association Review. 1995; 18(6):501-11.

21. Partinen $M$, Jamieson A, Guillleminault C. Long term outcome for obstructive sleep apnea syndrome patients. Mortality Chest. 1988;94:1200-4.

22. Robinson A, Guilleminault C. Obstructive sleep apnea syndrome. In: Chokroverty S. Sleep disorders Medicine: basic science, technical considerations, and clinical aspects. 2nd ed. Woburn: Butterworth Heinemann; 1999. p. 331-54.

23. Rogers RR, Lowe AA. Oral appliances for the management de snoring and obstructive sleep apnea. 2nd ed. Wexford: Sleep Disorders Dental Society; 1996.

24. Rose EC, Barthlen GM, Staats R, Jonas IE. Therapeutic efficacy of an oral appliance in the treatment of obstructive sleep apnea: a 2-year follow-up. Am J Orthod. 2002;121:273-9.

25. Sakakibara H, Tong M, Matsushita K, Hirata M, Konish $Y$, Suetsugu S. Cephalometric abnormalities in non-obese and obese patients with obstructive sleep apnoea. Eur Respir J. 1999;13:403-10.

26. Shamsuzzaman AS. Obstructive sleep apnea: implication for cardiac and vascular disease. JAMA. 2003;290(14):1906-14.

27. Smirne S, lannaccone S, Ferini-Strambi L. Muscle fiber type and habitual snoring. Lancet. 1991;337:597-9.

28. Sullivan CE, Issa FG, Berthon-Jones M, Eves L. Reversal of obstructive sleep apnea by continuous positive airway pressure. Lancet. 1981;1:862-5.

29. Tegelberg A, Walker-Engström ML, Vestling O, Wilhelmsson B Two different degrees of mandibular advancement with a dental appliance in treatment of patients with mild to moderate obstructive sleep apnea. Acta Odontol Scand. 2003;61(6):35662.

30. Young T, Palta M, Dempsey J, Skatrud J, Weber S, Badr S. The occurrence of sleep-disordered breathing among middle-aged adults. N Engl J Med. 1993;328:1230-5.
Endereço para correspondência

Cauby Maia Chaves Junior

Rua Tibúrcio Cavalcante, 2860, Dionísio Torres

CEP: 60.125-101 - Fortaleza / CE

E-mail: cmcjr@uol.com.br 